\title{
Calpain as a Therapeutic Target in Traumatic Brain Injury
}

\author{
Kathryn E. Saatman, ${ }^{*}$ Jennifer Creed ${ }^{\dagger}$ and Ramesh Raghupathi ${ }^{\dagger}$ \\ *Spinal Cord and Brain Injury Research Center, Department of Physiology, University of Kentucky, Lexington, Kentucky 40536-0509; \\ and ${ }^{\dagger}$ Department of Neurobiology and Anatomy, Drexel University College of Medicine, Philadelphia, Pennsylvania 19129
}

Summary: The family of calcium-activated neutral proteases, calpains, appears to play a key role in neuropathologic events following traumatic brain injury (TBI). Neuronal calpain activation has been observed within minutes to hours after either contusive or diffuse brain trauma in animals, suggesting that calpains are an early mediator of neuronal damage. Whereas transient calpain activation triggers numerous cell signaling and remodeling events involved in normal physiological processes, the sustained calpain activation produced by trauma is associated with neuron death and axonal degeneration in multiple models of TBI. Nonetheless, the causal relationship between calpain activation and neuronal death is not fully understood. Much remains to be learned regarding the endogenous regulatory mechanisms for controlling calpain activity, the roles of different calpain isoforms, and the in vivo substrates affected by calpain. Detection of stable proteolytic fragments of the submembrane cytoskeletal protein $\alpha$ II-spectrin specific for cleavage by calpains has been the most widely used marker of calpain activation in models of TBI. More recently, these protein fragments have been detected in the cerebrospinal fluid after TBI, driving interest in their potential utility as TBIassociated biomarkers. Post-traumatic inhibition of calpains, either direct or indirect through targets related to intracellular calcium regulation, is associated with attenuation of functional and behavioral deficits, axonal pathology, and cell death in animal models of TBI. This review focuses on the current state of knowledge of the role of calpains in TBI-induced neuropathology and effectiveness of calpain as a therapeutic target in the acute post-traumatic period. Key Words: Biomarker, calcium, neuroprotection, protease, proteolysis, spectrin.

\section{INTRODUCTION}

Calpains are nonlysosomal, neutral cysteine proteases activated by calcium. Of the several isoforms of calpains that appear to be expressed in the brain, ${ }^{1}$ the two most well characterized are the ubiquitous calcium-regulated isoforms $\mu$-calpain and $\mathrm{m}$-calpain. Each exists as a heterodimer comprised of a unique large $(80 \mathrm{kDa})$ catalytic subunit and a common small $(28 \mathrm{kDa})$ regulatory subunit. Both the large and small subunits contain multiple calcium binding sites. Elevations in intracellular free calcium above isoform-specific thresholds $(\sim 1 \mu \mathrm{mol} / \mathrm{L}$ for $\mu$-calpain and $\sim 1 \mathrm{mmol} / \mathrm{L}$ for $\mathrm{m}$-calpain) result in calcium binding and subsequent activation via a conformational change that creates an active catalytic triad, ${ }^{2}$ as well as autolysis of both subunits. Because $\mu$-calpain and m-calpain have essentially identical substrate specificity and virtually nothing is yet known of their differential

Address correspondence and reprint requests to: Kathryn E. Saatman, Ph.D., Spinal Cord and Brain Injury Research Center (SCoBIRC), University of Kentucky, B473 BBSRB, 741 S. Limestone St., Lexington, KY 40536-0509. E-mail: k.saatman@uky.edu. roles in trauma, this review will discuss calpains in general, referring to both isoforms.

The range of physiological functions of calpains is not yet fully appreciated, but transient calpain activation is involved in essential functions such as cell signaling, synaptic plasticity, and protein turnover. ${ }^{3,4}$ In contrast, sustained activation of calpains contributes to both chronic neurodegeneration associated with Parkinson's disease, Alzheimer's disease, and Huntington's disease ${ }^{5}$ and acute neuronal damage resulting from traumatic brain injury (TBI), ${ }^{6,7}$ stroke, ${ }^{1}$ hypoxia, ${ }^{8,9}$ and spinal cord injury. ${ }^{10}$ Calpains are also implicated in pathologies outside of the CNS, including muscular dystrophies, type 2 diabetes mellitus, cataract formation, and myocardial infarction. ${ }^{3}$ Although experimental evidence for the involvement of calpains in pathological states is strong, much remains to be learned about the regulation of calpain activity, the identity and relative importance of in vivo substrates, and differential roles and subcellular localization of calpain isoforms.

This review will discuss the current state of knowledge of calpain activation patterns in experimental models of TBI, in vivo calpain substrates, the relationship of 
calpain-mediated proteolysis to neuronal death, and the emerging role for calpain-specific proteolytic fragments as biomarkers in TBI. Finally, preclinical efforts at improving outcome after TBI through calpain inhibition will be reviewed.

\section{POST-TRAUMATIC CALPAIN ACTIVATION}

\section{Regional-temporal profile}

The first reports of calpain activation following experimental TBI were published in 1996. Using a combination of immunoblotting and immunohistochemical analyses for breakdown products (BDPs) of the submembrane cytoskeleton protein $\alpha \mathrm{II}$-spectrin specifically generated by calpains, Saatman et al. ${ }^{7}$ showed that lateral fluid percussion brain injury in the rat initiates calpain activation in neuronal dendrites of the cortex and hippocampus and in axons of damaged white matter tracts within $90 \mathrm{~min}$ after injury. By $4 \mathrm{~h}$ after injury, calpain activation was noted in neuronal somata as well, and by $24 \mathrm{~h}$ degenerating cortical and hippocampal neurons were intensely labeled for calpain-mediated spectrin BDPs (FIG. 1). Using another model of contusive brain injury in which dark, shrunken neurons can be observed
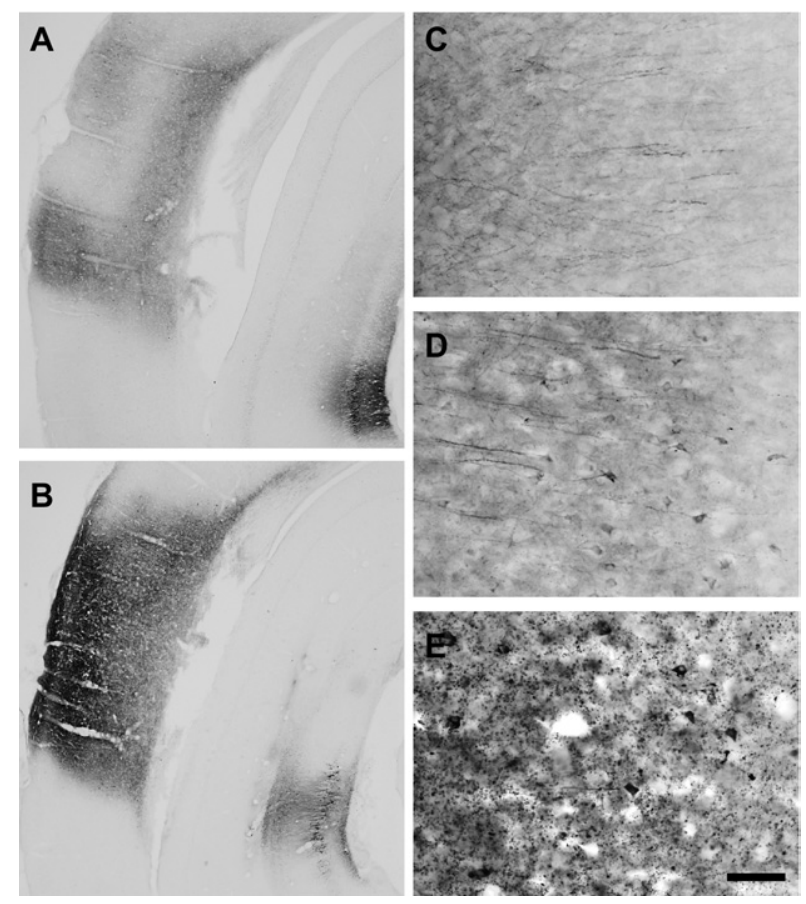

FIG. 1. Regional and subcellular localization of calpain-mediated spectrin breakdown after lateral fluid percussion brain injury in rats. Immunohistochemistry with an antibody specific for calpain-mediated proteolytic fragments of spectrin reveals a highly localized pattern of calpain activation in the contused cortex and $\mathrm{CA} 3$ region of the hippocampus in the hemisphere ipsilateral to injury that increases in intensity from $4 \mathrm{~h}(\mathrm{~A})$ to $24 \mathrm{~h}(\mathrm{~B})$ after injury. Within the contused cortex, neuronal labeling was first observed in dendrites at $90 \mathrm{~min}(\mathrm{C})$, in the dendrites and somata at $4 \mathrm{~h}(\mathrm{D})$, and in degenerating neurons at $24 \mathrm{~h}(\mathrm{E})$. Scale bar in (E) represents $50 \mu \mathrm{m}$ and applies also to (C) and (D). histopathologically within 15 min after TBI, Kampfl et al. ${ }^{6}$ demonstrated an even more rapid post-traumatic activation of calpains. Lateral controlled cortical impact (CCI) brain injury in the rat resulted in calpain-mediated spectrin BDPs in the injured cortex as early as $15 \mathrm{~min}$, with levels increasing up to $24 \mathrm{~h}$ after injury. Quantification of autolysis of the large subunit of $\mu$-calpain from $80 \mathrm{kDa}$ to $76 \mathrm{kDa}^{6}$ and of $\mu$-calpain and m-calpain activity using casein zymography ${ }^{11}$ both corroborated an early increase in calpain activity, from 15 min until $6 \mathrm{~h}$ after lateral $\mathrm{CCI}$ in the rat.

In addition to the commonly used rat TBI models of lateral fluid percussion and CCI described above, calpain activation (typically assayed using spectrin proteolysis as a surrogate marker for calpain activity) has been demonstrated in mouse models ${ }^{12-14}$ and in several other types of in vivo TBI models, including impact acceleration, weight drop, closed skull impact, midline fluid percussion, and optic nerve stretch, ${ }^{12,14-17}$ confirming that post-traumatic calpain activation is a common pathological event triggered by both focal and diffuse traumatic insults. Nonetheless, the time course of activation may differ with the type of brain injury sustained. For example, diffuse brain injury produced by weight drop to the closed skull results in a significant increase in the level of calpain-mediated $145-\mathrm{kDa}$ spectrin BDPs in the cortex, hippocampus, and striatum, but only after $24-48 \mathrm{~h}$ in male mice, ${ }^{14,18}$ whereas focal cortical contusion induced by CCI elicits increased cortical calpain-mediated proteolysis within minutes to several hours (FIG. 2). ${ }^{6,19,20}$ Injury severity has also been shown to affect calpain activation patterns in the hippocampus or in septohippocampal cultures. ${ }^{21-23}$ Little is known about potential sex-related differences in post-traumatic calpain activation, but Kupina et al. ${ }^{18}$ noted a less widespread and more delayed pattern of calpain activation in female, compared with male, mice subjected to diffuse brain injury.

Using trauma models that produce predominantly axonal pathology, calpain-mediated spectrin proteolysis was observed in axons within minutes to hours after injury. ${ }^{12,15,16}$ Electron microscopy revealed that spectrin BDPs were initially localized near the axolemma, but were distributed throughout the axon cylinder at later time points. ${ }^{15}$ In optic nerve axons of mice, stretch injury elicited a biphasic pattern of calpain activation, with an initial transient phase of proteolytic activity in intact axons and a second phase of calpain-mediated proteolysis on the order of days after injury in disconnected and degenerating axons. ${ }^{12}$ This second phase of calpain activation is likely involved in the Wallerian degeneration of damaged axons. Early calpain-mediated spectrin proteolysis in intact axons followed by the later appearance of punctate labeling for axonal spectrin BDPs, consistent with axonal degeneration, has also been observed after 


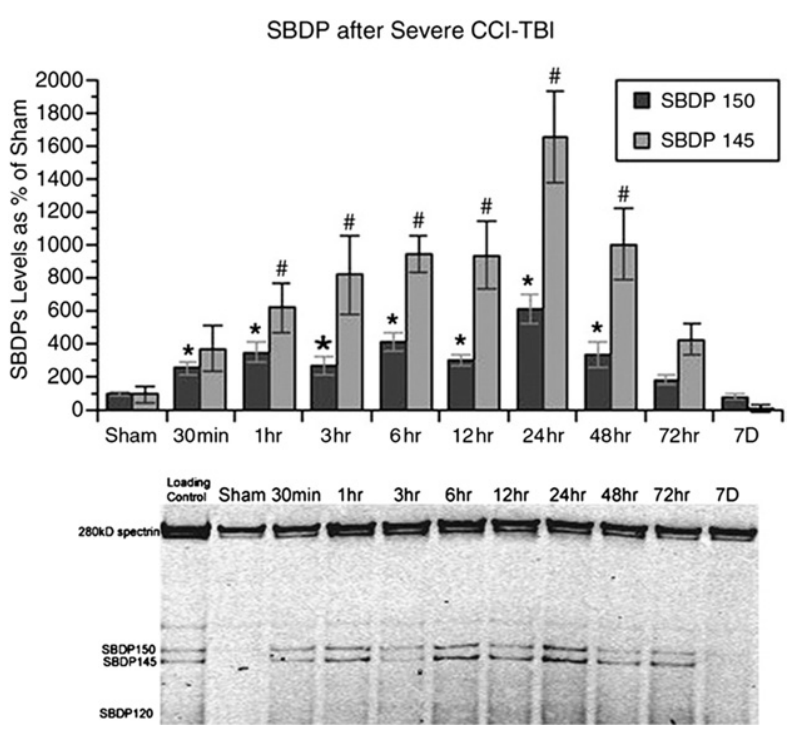

FIG. 2. Time course of spectrin proteolysis in the cortex after severe controlled cortical impact injury in mice. Immunoblotting for spectrin revealed a progressive increase in the $150-\mathrm{kDa}$ spectrin breakdown product (SBDP) and the calpain-specific $145-\mathrm{kDa}$ SBDP, beginning at $30 \mathrm{~min}$ and $1 \mathrm{~h}$, respectively. Significantly increased spectrin breakdown was observed through $48 \mathrm{~h}$ after injury. $N=8$ animals per time point. Data are presented as means; error bars indicate standard error. One-way analysis of variance with post hoc Fisher's protected least significant difference tests: * $p<0.0001$ vs. sham SBDP 150; \# $p<$ 0.0001 vs. sham SBDP145. Reprinted from Deng et al., ${ }^{20}$ with permission.

closed head injury in 17 -day-old rats. ${ }^{17}$ The clinical relevance of post-traumatic axonal calpain activation was corroborated by a study in which both levels of calpainmediated spectrin BDPs and the ratio of autolyzed to nonautolyzed large subunit of m-calpain $(76 \mathrm{kDa} / 80$ $\mathrm{kDa}$ ) were shown to be elevated in the corpus callosum of humans who had died after blunt head trauma and showed postmortem evidence of diffuse axonal injury. ${ }^{24}$

Calpain activation is involved in the pathology of traumatic axonal injury, but the nature of the role of calpains may depend on a multiplicity of factors, many of which are not yet understood. In models of contusive TBI, calpain activation in white matter may play a relatively minor role, ${ }^{17,25}$ whereas it may be much more instrumental in models of diffuse brain injury that mimic clinical diffuse axonal injury. ${ }^{15,16}$ In addition, the extent of calpain-mediated proteolysis in axons may be highly dependent on injury severity or the number of insults. ${ }^{26}$ Calpain-mediated spectrin BDPs have been colocalized with compacted neurofilaments in damaged axons, ${ }^{15}$ but spectrin degradation by calpains does not always correspond temporally or spatially to other well-established markers of axonal injury, such as the accumulation of $\beta$-amyloid precursor protein ${ }^{17}$ or neurofilament protein. ${ }^{12}$ These data highlight the complexity of axonal injury and the need for a greater understanding of axonal substrates and regulation of calpains.

\section{Regulation}

Intracellular free calcium is the primary regulator of calpain activation, but calpains are also regulated through interaction with a specific inhibitor protein, calpastatin. In the presence of calcium, one calpastatin molecule can bind to and inhibit the proteolytic activity of four molecules of calpain. ${ }^{27}$ Calpains and calpastatin appear to be coexpressed in all cell types of the CNS. Some studies, however, suggest that these proteins may not be exclusively cytosolic, ${ }^{28-30}$ raising the possibility that the extent of inhibition of calpains by endogenous calpastatin may vary according to subcellular localization. Experimental evidence suggests that calpain activity is greater near individual mitochondria ${ }^{15}$ or accumulations of mitochondria ${ }^{31}$ in traumatically injured axons. The intracellular localization of calpains and calpastatin may also change as a result of injury, in that calcium increases have been shown to trigger translocation of calpains to the plasma membrane ${ }^{3}$ and shifts in intracellular calpastatin pools. ${ }^{32}$ After TBI in rats, a relative shift in $\mu$-calpain activity from the cytosol to the total membrane fraction was observed from 3 to $24 \mathrm{~h}$ after injury, ${ }^{11}$ a time frame consistent with increased calpain autolysis and proteolytic activity. ${ }^{6}$

Little is known about the response of calpastatin to TBI or about its effectiveness in inhibiting post-traumatic calpain activation or its downstream consequences. In rats, CCI brain injury elicited a delayed increase in an 80-kDa calpastatin-positive band on immunoblots, relative to a $130-\mathrm{kDa}$ band. ${ }^{33}$ It is unclear whether the increase in the $80-\mathrm{kDa}$ band represented increased expression of a calpastatin isoform ${ }^{34}$ or cleavage of a higher molecular weight isoform. Mice deficient in calpastatin exhibit greater motor dysfunction and a more pronounced memory deficit than wild-type mice after CCI brain injury, but showed no difference in their volume of cortical tissue damage. ${ }^{35}$ These data suggest that endogenous calpastatin plays a role in limiting behavioral dysfunction after TBI, but do not support a neuroprotective effect in neurons within a focal contusion.

Other means of regulating calpain activity include transcriptional regulation, phosphorylation, and oxidation. Cortical and hippocampal $\mu$-calpain mRNA levels increase in a delayed fashion, at $72 \mathrm{~h}$ after CCI in rats, and are preceded by increased $\mathrm{m}$-calpain and calpastatin mRNA at $24 \mathrm{~h} .{ }^{36}$ Although calpains and calpastatin have multiple phosphorylation sites, ${ }^{3}$ no published studies have examined the effect of phosphorylation on posttraumatic calpain activity. Oxidation inhibits proteolytic activity, but not autolysis, of calpains. ${ }^{37}$ Given that free radical generation is a well-established acute consequence of TBI, ${ }^{38}$ oxidative damage may modulate calpain function in the injured brain. 


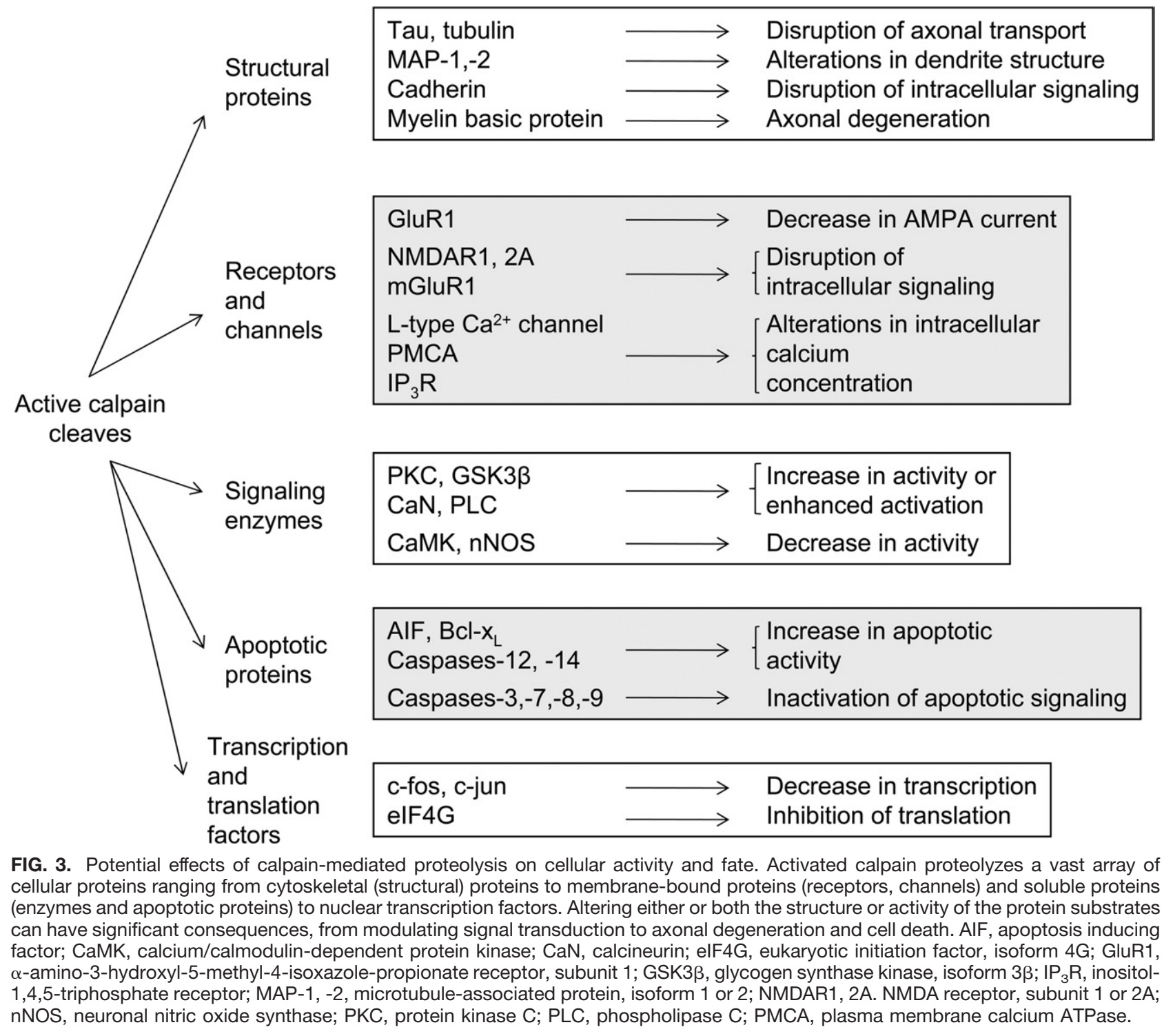

\section{Potential cellular substrates}

The calpain substrate most widely studied in models of TBI is the $280-\mathrm{kDa}$ nonerythroid $\alpha \mathrm{II}$-spectrin protein, which provides structural support to the plasma membrane cytoskeleton, particularly in axons and presynaptic terminals. The identification of $\alpha$ II-spectrin cleavage sites unique to calpains enabled the development of cleavage-specific antibodies suitable for use in immunohistochemistry and immunoblotting. ${ }^{39,40}$ Furthermore, whereas calpain-mediated proteolysis of $\alpha$ II-spectrin results in stable $150-\mathrm{kDa}$ and $145-\mathrm{kDa}$ fragments, caspase-3 mediated cleavage results in $150-\mathrm{kDa}$ and $120-$ $\mathrm{kDa}$ fragments, allowing for the differential detection of calpain and caspase- 3 activation on immunoblots of tissue or cell homogenates, serum, or cerebrospinal fluid (CSF). ${ }^{41}$ Although detection of calpain-generated spectrin BDPs has provided a powerful and widely applicable tool for the study of the role of calpains in neurodegen- erative disease and injury in vivo, a more complete understanding of the functional consequences of post-traumatic calpain activation requires the identification of a much broader spectrum of in vivo calpain substrates.

In studies using purified proteins or cell culture assays, calpains have been shown to cleave a very large number of cellular substrates encompassing a host of cytoskeletal proteins, signal transduction proteins, membrane-associated proteins, neurotransmitters, and transcription factors (FIG. 3). ${ }^{1,3}$ Although some putative calpain substrate proteins (e.g., microtubule-associated protein 2, growthassociated protein- 43 , and Bid) have been shown to be cleaved or decreased in level after experimental $\mathrm{TBI},{ }^{21,42,43}$ a specific role for calpain in post-traumatic proteolysis has been difficult to establish, for lack of truly specific calpain inhibitors and calpain-specific cleavage markers. Because most calpain substrate proteins are susceptible to degradation by several other pro- 
teases, such as cathepsins or caspases, relationships between post-traumatic calpain activation and the loss of putative calpain substrates must be interpreted cautiously in in vivo trauma models, in which the environment cannot be manipulated to the extent that it can in vitro. In vitro models of neurotrauma can be used to test effects of multiple inhibitors, overexpression of calpastatin, and manipulations of extracellular and intracellular calcium to more definitively identify calpain substrates. Recently, trauma-induced cleavage of voltage-gated sodium channels by calpain was described using this approach. ${ }^{44}$

The neurofilament triplet proteins NF68, NF160, and NF200 are known calpain substrates in vitro, and TBI induces loss of neurofilament proteins in gray and white matter regions. ${ }^{18,24,45-48}$ A role for calpains in neurofilament degradation is supported by evidence that NF68 BDPs detected after contusive injury are similar in molecular weight to those generated by in vitro calpain digestion, ${ }^{49}$ and that calpain inhibitors administered to brain-injured animals attenuate the loss of NF68 and NF200. ${ }^{50}$ More recently, four isoforms of myelin basic protein (MBP) were shown to be degraded after CCI brain injury in rats, resulting in stable BDPs. ${ }^{51}$ An antibody against the $\mathrm{N}$-terminus of these proteolytic fragments, obtained from brain-injured mice, also detected MBP fragments of an identical molecular weight produced by incubating either naïve homogenates or purified MBP with calpain. These fragments were not observed after incubation with caspase-3, cathepsin B or D, or matrix metalloproteinase- 2 or -9 , providing strong support for an in vivo role for calpains in the proteolysis of MBP after TBI.

In a study using a similar approach of matching the molecular weights of BDPs identified after TBI to those produced by incubation of naïve brain homogenates with activated calpain, collapsin response mediator protein 2 (CRMP-2) was implicated as a potential calpain substrate after CCI brain injury in rats. ${ }^{52}$ Calpain-mediated truncation of CRMPs after TBI may have implications for axonal sprouting or growth, axonal degeneration, and neuronal death. ${ }^{52}$

This approach is being expanded using proteomic or degradomic techniques, examining large numbers of proteins from serum or CSF samples from brain-injured humans or brain tissue obtained from brain-injured animals for either decreases in intact protein or the appearance of novel low molecular weight proteins that could be putative BDPs. In a study using a high-throughput immunoblotting technique, 40 proteins downregulated in the hippocampus at $48 \mathrm{~h}$ after severe CCI in rat were identical to those degraded after incubation with m-calpain, suggesting that they may be targets for calpain after TBI. ${ }^{53}$ An alternate approach for identifying calpain substrates is to screen proteolytic patterns from in vitro models of cell death known to involve calpains and then look for selected BDPs in an in vivo model of TBI. ${ }^{54}$

\section{Relationship to neurodegeneration}

Although calpain-mediated proteolysis of spectrin after TBI is well established, the significance of this event in the context of cell death is more ambiguous. Evidence that ties neurodegeneration after trauma to calpain activation stems from work both in vitro and in vivo. The in vivo models of $\mathrm{TBI}$, for the most part, support a close correspondence between areas exhibiting persistent calpain activation and those displaying foci of neuronal degeneration. For example, in both lateral fluid percussion and lateral CCI models, calpain-mediated spectrin BDPs were localized ipsilateral to the percussion or impact injury, and were absent from the contralateral hemisphere. ${ }^{6,7,19}$ Furthermore, comparisons of patterns of calpain-mediated spectrin fragments and histological stains reveal that the location and timing of calpain proteolytic activity is coincident with the loss of histological stain and with morphological changes such as dendritic beading or fragmentation or somal shrinkage or blebbing. $6,7,25$

In the hippocampus, calpain activation was restricted to the CA3 region, ${ }^{7}$ an area of selective neuronal loss in lateral fluid percussion brain injury. ${ }^{55}$ Notably, thalamic neurons, remote from the contusion site, display delayed alterations in calcium flux, ${ }^{56}$ calpain activation, ${ }^{7}$ and cell death. ${ }^{55}$ Time-course studies suggest that the peak in post-traumatic $\mu$-calpain autolysis precedes the peak accumulation of calpain-mediated spectrin BDPs, ${ }^{6}$ which in turn precedes the peak of neurodegeneration. ${ }^{57}$ In septohippocampal cultures, mechanical stretch injury capable of evoking a persistent elevation in calpain-mediated spectrin BDPs also induced the release of cytosolic lactate dehydrogenase and uptake of nuclear propidium iodide, two commonly used indicators of cell death. Neurons in these injured cultures also displayed fragmented and beaded dendrites, loss of NeuN immunostaining, and nuclear changes consistent with necrotic or apoptotic cell death. ${ }^{23}$ Together, these in vivo and in vitro data support the prevailing hypothesis that prolonged calpain activation contributes to post-traumatic cell death.

Nevertheless, sublethal calpain activation may also occur, perhaps contributing to transient cellular damage or adaptive responses. For example, neuronal cultures subjected to mild stretch injury, which resulted in a transient elevation in spectrin BDPs, showed no significant neuronal death. ${ }^{23}$ In vivo, calpain-mediated spectrin BDPs have been observed in the hippocampus ipsilateral to CCI injury in the absence of overt hippocampal cell death, suggesting that calpain activation may occur in the absence of extensive morphopathological changes. ${ }^{25}$

In an elegant study using tracer molecules introduced into the extracellular space before and after TBI, Farkas 
et al. ${ }^{58}$ demonstrated that calpain-mediated spectrin BDPs were not typically localized in neurons exhibiting plasma membrane compromise or necrosis. Rather, the majority of neurons labeled for spectrin BDPs displayed only limited ultrastructural change. After lateral CCI brain injury, both $\mu$ - and m-calpain activity increased in the contralateral cortices, ${ }^{11}$ despite a lack of calpainmediated spectrin proteolysis detected by immunoblotting or overt neuron loss. ${ }^{6,19}$ It is possible, however, that calpain activation in the contralateral hemisphere contributes to, or is reflective of, diffuse axonal degeneration. $^{13}$

Trauma may also initiate cell death without substantial involvement of calpains. In the lateral fluid percussion model of TBI, neurons of the hippocampal dentate hilus degenerate early, ${ }^{55}$ even after mild injury, ${ }^{59}$ but do not exhibit calpain-mediated spectrin proteolysis, ${ }^{7}$ suggesting that hilar neuron death was independent of calpains. Diffuse TBI leads to membrane compromise and necrotic change in cortical neurons, most often in the absence of calpain-mediated spectrin degradation. ${ }^{58} \mathrm{Me}-$ chanical stretch injury to organotypic hippocampal slices results in calpain activation and caspase- 3 activation in distinct subpopulations of neurons, with no appreciable colocalization. $^{22}$

Colocalization studies have not been performed in in vivo models of TBI, but the temporal patterns of caspase- 3 and calpain activation are reasonably different. Caspase- 3 activation is detected in the cortex and hippocampus at $8-12$ hours after injury, peaks at 24 hours, and is substantially reduced by 3 days after injury, ${ }^{19,60-62}$ whereas calpain activation occurs earlier (by 3-4 hours) and is sustained up to 7 days after injury. ${ }^{7,19}$ Therefore, given that TBI is known to initiate caspase-3 activation and apoptotic cell death, ${ }^{63}$ it is likely that a subset of damaged neurons will die via apoptosis without calpain activation.

Collectively, these various studies suggest that spectrin proteolysis by calpain is not necessary or sufficient for neuron death after TBI, and emphasize the need for caution in interpreting the occurrence of calpain-mediated spectrin breakdown and its overall implications for neuronal injury and death.

\section{CALPAIN-SPECIFIC BREAKDOWN PRODUCTS AS BIOMARKERS IN TBI}

Biomarkers are desirable in clinical settings to improve diagnosis or prognosis for head-injured patients, improve sensitivity of detection of mild TBI, predict adverse events associated with TBI, or select patients with given pathophysiologic characteristics for treatment. ${ }^{64,65}$ Clinically useful biomarkers might be measured in brain microdialysate, CSF, or serum. Ideally, TBI biomarkers should be specific to brain trauma, and their levels should vary with injury severity and correlate with functional or histological outcome. Calpain-mediated BDPs make good candidates for biomarkers because they are absent or present at low levels in uninjured animals or humans, increasing the signal-to-noise ratio. Furthermore, they are linked to a pathophysiologic event (i.e., prolonged activation of calpains) that is critical in neurodegeneration.

To date, efforts at characterizing a biomarker for TBI related to calpain have focused on $\alpha \mathrm{II}$-spectrin, although other proteins with stable calpain-generated fragments may be candidates as well. ${ }^{66}$ In either adult or immature animals, levels of spectrin BDPs increase in both brain tissue and CSF within the first $72 \mathrm{~h}$ after TBI. ${ }^{67,68}$ Trauma-induced increases in CSF spectrin BDP levels were proportional to injury severity ${ }^{54,69}$ but did not correlate with lesion size after contusive injury. ${ }^{69}$ Together with recent data demonstrating that concussive TBI in rats was associated with increased CSF levels of calpainmediated spectrin BDPs, ${ }^{16}$ these observations suggest that both contusive damage and axonal injury may contribute to the appearance of spectrin BDPs in the CSF of brain-injured animals.

Several prospective case-control studies of patients with severe TBI have reported increased levels of calpain-mediated and caspase-mediated spectrin BDPs in ventricular CSF from $6 \mathrm{~h}$ to $3-4$ days after trauma. ${ }^{70-73}$ Although TBI is often associated with elevated intracranial pressure, severe TBI patients had significantly higher CSF levels of spectrin and spectrin BDPs than did patients with elevated intracranial pressure from nontraumatic causes. ${ }^{70,73}$ Higher levels of the $145-\mathrm{kDa}$ spectrin fragment were measured in CSF of those patients with a Glasgow Coma Scale of 3-5, compared with patients with a score of $6-8,{ }^{71}$ indicating a relationship with injury severity. Acute elevations in CSF levels of spectrin BDPs were higher in patients with declines in Glasgow Coma Scale over the first $24 \mathrm{~h}$ after head injury ${ }^{73}$ and in those with poor outcome at 6 months. ${ }^{71}$ Furthermore, persistent elevations in intact spectrin and spectrin BDPs in CSF were associated with a worse outcome at 6 months after discharge, compared with the outcome for patients with decreasing CSF levels over the first 4 days after TBI. ${ }^{72}$

Identification and characterization of biomarkers for TBI is a relatively young field of research, and is still rapidly evolving. To date, animal model and clinical TBI studies suggest that calpain-mediated spectrin BDPs may provide a reliable marker of brain injury, detectable in brain tissue or CSF. Initial studies provide promising data to suggest that analyses of the levels, duration, and rate of change of concentrations of spectrin or spectrin fragments may provide important information regarding injury severity, clinical course, or outcome. Consequently, markers of calpain-mediated proteolysis may 


\section{Calpain Inhibitor II}<smiles>CCC(NC(=O)C(CC(C)C)NC(C)=O)C(=O)NC(C=O)C(=O)O</smiles>

\section{SJA6017}<smiles>CC(C)CC(C=O)NC(=O)C(NS(=O)(=O)c1ccc(F)cc1)C(C)C</smiles>

AK295<smiles>CCC(NC(=O)C(CC(C)C)NC(=O)OCc1ccccc1)C(=O)NCCCN1CCOCC1</smiles>

MDL-28170 (Calpain Inhibitor III)<smiles>CC(C)C(NC(=O)OCc1ccccc1)C(=O)NC(C=O)Cc1ccccc1</smiles><smiles>CCCCC(CC(N)=O)NC(=O)c1ccccc1/C=C/c1ccc(CN(C)C)cc1</smiles>

FIG. 4. Chemical structures for calpain inhibitors tested in animal models of traumatic brain injury. In vivo studies (discussed in the text) of calpain inhibitor II, AK295, SJA6017, MDL-28170 (calpain inhibitor III), and calpain inhibitor 5b have demonstrated efficacy in selected outcomes.

serve as important tools in future clinical trials, to aid in stratification of patients for treatment or in early assessment of treatment efficacy. A recent study demonstrated that pretreatment with the M1-specific muscarinic cholinergic receptor antagonist dicyclomine attenuated 145k-Da spectrin BDP levels in the CSF at $24 \mathrm{~h}$ after moderate lateral fluid percussion in rats. ${ }^{74}$ It remains to be determined whether decreasing CSF concentrations of calpain-mediated spectrin BDPs by means of this or other treatments leads to improved outcome.

\section{THERAPEUTIC POTENTIAL OF POST- TRAUMATIC CALPAIN INHIBITION}

Given the correlation between prolonged calpain activation and trauma-induced neurodegeneration and the broad range of protein targets that could potentially be protected, calpain inhibition is an attractive therapeutic approach. Because basal levels of calpain activity in the brain are relatively low, short-term inhibition of calpains may have fewer side effects than strategies to antagonize other common targets, such as glutamate receptors. The goal of calpain inhibition as a treatment strategy for TBI is to prevent or reduce degradation of multiple neuronal or nonneuronal substrate proteins, such as cytoskeletal proteins and signaling molecules, thereby attenuating cell death and improving functional outcome. In evaluating a therapy, it is important to consider 1) the need for improving functional recovery as well as structural integrity after injury and 2) the appropriate dose and therapeutic time window. Calpains may be inhibited directly, using specific calpain inhibitors or more broadly acting protease inhibitors, or indirectly, via other targets that modulate intracellular free calcium levels.

\section{Calpain and cysteine protease inhibitors}

A handful of studies have evaluated calpain inhibitors in experimental models of TBI. The chemical structures of these inhibitors are illustrated in FIG. 4. Calpain inhibitor II ( $N$-acetyl-leu-leu-methioninal), when administered by continuous intra-arterial infusion for $24 \mathrm{~h}$ after cortical impact injury in the rat, significantly reduced 
loss of NF68 and NF200 and calpain-mediated spectrin proteolysis in the cortex, suggesting cytoskeletal protection. ${ }^{50}$ A subsequent study, however, found that calpain inhibitor II given at $5 \mathrm{~min}$ and $6 \mathrm{~h}$ after weight-drop brain injury in the rat failed to attenuate loss of microtubuleassociated protein $2 .{ }^{75}$ Because calpain inhibitor II is not selective for calpains over certain cathepsins $\left(K_{\mathrm{i}}\right.$ for $\mu$-calpain $=120 \mathrm{nmol} / \mathrm{L}, K_{\mathrm{i}}$ for m-calpain $=230$ $\mathrm{nmol} / \mathrm{L}, K_{\mathrm{i}}$ for cathepsin B $=100 \mathrm{nmol} / \mathrm{L}$, and $K_{\mathrm{i}}$ for cathepsin $\mathrm{L}=600 \mathrm{pmol} / \mathrm{L}$ ), interpretation of the role of calpain inhibition in these studies is not straightforward.

Post-traumatic administration of the ketoamide calpain inhibitor AK295 [Z-Leu-aminobutyric acid-CONH $\left(\mathrm{CH}_{2}\right)_{3}$ morpholine], given by continuous intra-arterial infusion for $48 \mathrm{~h}$, significantly attenuated motor and memory impairment at 1 week after lateral fluid percussion brain injury in rats, implicating calpains in post-traumatic events underlying neuromotor and cognitive dysfunction. ${ }^{76}$ Surprisingly, although this reversible calpain inhibitor is relatively selective for calpains $\left(K_{\mathrm{i}}=27-42\right.$ $\mathrm{nmol} / \mathrm{L}$ ) over other cysteine proteases such as cathepsin B $\left(K_{\mathrm{i}}=24 \mu \mathrm{mol} / \mathrm{L}\right)$, administration of AK295 did not reduce calpain-mediated spectrin BDPs in the cortex or hippocampus at $48 \mathrm{~h}$ or 1 week after TBI ${ }^{77}$ Furthermore, cortical lesion size and the number of apoptotic cells in the cortex, subcortical white matter, and hippocampus were equivalent in rats receiving AK295 and saline vehicle, suggesting that overt neuroprotection was not required for the behavioral improvements elicited by AK295.

A similar functional improvement without protection of spectrin was observed in a study by Kupina et al. ${ }^{14}$ using the reversible peptide aldehyde calpain inhibitor SJA6017. Mice subjected to diffuse weight-drop brain injury showed a dose-dependent improvement in motor function, even with intravenous administration of SJA6017 delayed up to $4 \mathrm{~h}$ after injury. The drug did not, however, reduce spectrin breakdown in cortical or hippocampal tissues.

Together, these studies support the hypothesis that prolonged calpain activation contributes to post-traumatic morbidity; however, they also reinforce the need to understand more fully the in vivo actions of calpains in the traumatically injured brain.

Calpain inhibitors may contribute to functional recovery by protecting against cell death in select populations of neurons or by reducing axonal or dendritic pathology. For example, improved neuronal survival in the dentate hilus at 2 weeks after injury has been reported with intraperitoneal injection of the nonpeptidic calpain inhibitor $5 \mathrm{~b}$ [N-(1-carbamoyl-1-oxohex-1-yl)-2-[E-2-(4-dimethylaminomethylphenyl)-ethen-1-yl]benzamide] at 15 min and $2 \mathrm{~h}$ after lateral fluid percussion brain injury in rats. ${ }^{78}$ Protection of white matter in TBI has been dem- onstrated using perhaps the most widely studied calpain inhibitor, MDL-28170, a cell permeable, reversible, peptidic aldehyde inhibitor of both $\mu$ - and m-calpain $\left(K_{\mathrm{i}}=\right.$ $10 \mathrm{nmol} / \mathrm{L} ; K_{\mathrm{i}}$ for cathepsin B $\left.=25 \mathrm{nmol} / \mathrm{L}\right) .{ }^{79}$ Pretreatment with a single intravenous bolus of MDL-28170, also known as calpain inhibitor III, reduced acute pathology associated with traumatic axonal injury (accumulation of amyloid precursor protein and neurofilament compaction) at $2 \mathrm{~h}$ after impact acceleration brain injury in rats. ${ }^{80}$ Preservation of injured white matter was also demonstrated in a subsequent study that explored functional and structural outcomes as well as therapeutic window for treatment. After midline fluid percussion brain injury in rats, MDL-28170 was effective in reducing amyloid precursor protein accumulation with up to a $4 \mathrm{~h}$ delay in initiating a single bolus treatment, but attenuation of injury-induced decreases in compound action potentials in the corpus callosum required treatment within 30 min after injury. ${ }^{81}$ Furthermore, structural and functional axonal protection were sustained for as long as 2 weeks if an initial 30-min bolus was coupled with $24 \mathrm{~h}$ and $48 \mathrm{~h}$ dosing, consistent with studies reporting prolonged calpain activation after TBI. ${ }^{6,7,14}$

\section{Indirect calpain inhibition}

Although the use of specific calpain inhibitors provides a direct means to preventing undesirable proteolysis after TBI, preventing activation might also be achieved at an earlier step by antagonizing a major entry route for ionic calcium in neurons: the N-methylD-aspartate (NMDA) subtype glutamate receptor. Using a stretch injury model in hippocampal slice cultures, DeRidder et al. ${ }^{22}$ have shown that, at moderate and severe injury levels, calpain is activated in the pyramidal cell layer of areas CA3 and CA4 and in the granule cell layer of the dentate gyrus. Pretreatment with NR2Bspecific or broad-spectrum NMDA receptor antagonists, but not an NR2A-specific antagonist, significantly reduced calpain-mediated spectrin proteolysis in these regions. These data suggest that NR2B-containing NMDA receptors play a key role in post-traumatic calpain activation, an insight that provides a new approach for more selective NMDA receptor antagonist therapies. Because both the NR2A and NR2B subunits can be cleaved by calpains, ${ }^{82}$ the interplay between calpain activation and NMDA receptor expression and function in the setting of TBI may be complex. In this regard, we have observed that, paradoxically, administration of the NR2B-selective antagonist before and after CCI significantly increases TBI-induced accumulation of spectrin BDPs (J. Creed et al., unpublished observations).

Magnesium modulates the activity of the NMDA receptor, creating a voltage-dependent block that inhibits $\mathrm{Ca}^{2+}$ influx. Traumatic injury results in widespread neuronal depolarization, thereby relieving the magnesium 
block of NMDA receptors. Supplementation with $\mathrm{Mg}^{2+}$ would be expected to partially preserve the blockade of the NMDA receptor, whereas a deficiency in available $\mathrm{Mg}^{2+}$ could exacerbate calcium influx after trauma. Consistent with this hypothesis, acute postinjury treatment with $\mathrm{Mg}^{2+}$ reduced calpain activation in the cortex and hippocampus of brain-injured rats, and also attenuated cortical tissue damage and MAP2 alterations. ${ }^{83}$ Conversely, prolonged dietary magnesium deficiency prior to injury enhanced calpain activity and worsened outcome. ${ }^{83}$ Therefore, although $\mathrm{Mg}^{2+}$ has a multiplicity of actions within the CNS, it may influence post-traumatic cell death and cytoskeletal disruption, in part, by modulating calpain activity.

Hypothermia is another treatment approach for TBI with numerous possible mechanisms of action. ${ }^{84}$ Hypothermia $\left(32^{\circ} \mathrm{C}\right)$ for $90 \mathrm{~min}$, with or without 90 additional minutes of rewarming, reduced acute calpain-mediated spectrin proteolysis in axons after impact acceleration brain injury in the rat. ${ }^{85}$ In clinical trials, however, magnesium sulfate ${ }^{86}$ and hypothermia ${ }^{87}$ in TBI failed to show beneficial effects.

Traumatic neuronal injury results in increased nonspecific plasma membrane permeability, ${ }^{88}$ which may allow an excessive influx of calcium sufficient to activate calpains. Although damage to the plasma membrane led to calpain activation only in a subset of neurons after diffuse brain injury in rats, ${ }^{58}$ axonal intracellular free calcium increases and calpain activation could be attenuated by extracellular calcium chelation or treatment with the amphiphilic copolymer poloxamer 188 to reseal membranes in neuronal cultures subjected to traumatic shear stress. ${ }^{31}$ Post-traumatic oxidative damage may exacerbate membrane damage and contribute to mitochondrial dysfunction, thereby enhancing neuronal calcium dysregulation. ${ }^{38}$ Repeated administration of the peroxynitrite-derived free radical scavenger, Tempol, beginning at $15 \mathrm{~min}$ after CCI in mice reduced calpain-mediated spectrin proteolysis at $24 \mathrm{~h}$, motor dysfunction at 2 days, and regional neurodegeneration at 7 days. ${ }^{89}$

The efficacy of immunophilin ligands to attenuate calpain activity after TBI raises the interesting possibility that inhibition of mitochondrial permeability transition may be an alternative means for reducing calpain-mediated proteolytic damage. For example, central administration of the immunophilin ligand cyclosporin A either before $^{90}$ or $30 \mathrm{~min}$ after $^{91}$ impact acceleration injury attenuated axonal calpain activation and neurofilament alterations in rats. Although cyclosporin A inhibits calcineurin as well as mitochondrial permeability transition, both cyclosporin A and NIM811 (a cyclosporin A analog that lacks the calcineurin-inhibitory capacity of cyclosporin A) reduced cortical calpain-mediated spectrin proteolysis, neurodegeneration, and motor dysfunction when given 15 min after severe CCI in mice. ${ }^{92}$ Admin- istration of the neuroimmunophilin ligand V-10,367 at $24 \mathrm{~h}$ after weight-drop head injury in mice resulted in attenuation of calpain-mediated spectrin proteolysis in the striatum at 3 days, ${ }^{93}$ suggesting a prolonged therapeutic window for influencing calpain-dependent pathology.

\section{CONCLUSIONS}

Calpain-mediated spectrin proteolysis has been extensively evaluated in animal and in vitro models of TBI as a surrogate marker of calpain activity. Acute, sustained calpain activation has been observed in many populations of vulnerable neurons, localized to multiple neuronal compartments, suggesting that calpains may be early mediators of neuronal dysfunction or death. Nonetheless, the relationship between calpain-mediated spectrin degradation and cell death is not straightforward, and may depend on neuronal subtype and injury mechanics, and on subcellular location, rate, and duration of calpain activation, as well as other factors. Calpain cleavage of other substrates, of which much less is known in the setting of TBI, may be more critical in determining the functional consequences of post-traumatic calpain activation. Stable BDPs generated after calpain cleavage of spectrin are detectable in the CSF of brain-injured animals and humans, and may serve as a useful biomarker of injury and response to treatment. Post-traumatic inhibition of calpains, either directly or indirectly, has been associated with attenuation of protein substrate degradation, neuronal damage, and behavioral deficits, fueling continued interest in calpain inhibition as a therapeutic approach in TBI. Despite substantial advances, much remains to be learned regarding the mechanisms by which calpain regulates cellular responses to traumatic injury.

Acknowledgments: This work was supported by National Institutes of Health grants NS058484 (K.E.S.) and HD41699 (R.R.), Kentucky Spinal Cord and Head Injury Research grants 6-12 and 7-10 (K.E.S.), and a Veterans Administration Merit Review grant (R.R.). We thank Dr. Edward D. Hall for preparation of Figure 4, and Carey Shaner for assistance in manuscript preparation.

\section{REFERENCES}

1. Bevers MB, Neumar RW. Mechanistic role of calpains in postischemic neurodegeneration. J Cereb Blood Flow Metab 2008;28: 655-673.

2. Hosfield CM, Elce JS, Davies PL, Jia Z. Crystal structure of calpain reveals the structural basis for $\mathrm{Ca}^{2+}$-dependent protease activity and a novel mode of enzyme activation. EMBO J 1999; 18:6880-6889.

3. Goll DE, Thompson VF, Li H, Wei W, Cong J. The calpain system. Physiol Rev 2003;83:731-801.

4. Wu HY, Lynch DR. Calpain and synaptic function. Mol Neurobiol 2006;33:215-236.

5. Vosler PS, Brennan CS, Chen J. Calpain-mediated signaling mech- 
anisms in neuronal injury and neurodegeneration. Mol Neurobiol 2008;38:78-100.

6. Kampfl A, Posmantur R, Nixon R, et al. mu-calpain activation and calpain-mediated cytoskeletal proteolysis following traumatic brain injury. J Neurochem 1996;67:1575-1583.

7. Saatman KE, Bozyczko-Coyne D, Marcy V, Siman R, McIntosh TK. Prolonged calpain-mediated spectrin breakdown occurs regionally following experimental brain injury in the rat. J Neuropathol Exp Neurol 1996;55:850-860.

8. Arai A, Vanderklish P, Kessler M, Lee K, Lynch G. A brief period of hypoxia causes proteolysis of cytoskeletal proteins in hippocampal slices. Brain Res 1991;555:276-280.

9. Hiramatsu K, Kassell NF, Lee KS. Improved posthypoxic recovery of synaptic transmission in gerbil neocortical slices treated with a calpain inhibitor. Stroke 1993;24:1725-1728.

10. Ray SK, Hogan EL, Banik NL. Calpain in the pathophysiology of spinal cord injury: neuroprotection with calpain inhibitors. Brain Res Brain Res Rev 2003;42:169-185.

11. Zhao X, Posmantur R, Kampfl A, et al. Subcellular localization and duration of mu-calpain and m-calpain activity after traumatic brain injury in the rat: a casein zymography study. J Cereb Blood Flow Metab 1998;18:161-167.

12. Saatman KE, Abai B, Grosvenor A, Vorwerk CK, Smith DH, Meaney DF. Traumatic axonal injury results in biphasic calpain activation and retrograde transport impairment in mice. J Cereb Blood Flow Metab 2003;23:34-42.

13. Hall ED, Sullivan PG, Gibson TR, Pavel KM, Thompson BM, Scheff SW. Spatial and temporal characteristics of neurodegeneration after controlled cortical impact in mice: more than a focal brain injury. J Neurotrauma 2005;22:252-265.

14. Kupina NC, Nath R, Bernath EE, et al. The novel calpain inhibitor SJA6017 improves functional outcome after delayed administration in a mouse model of diffuse brain injury. J Neurotrauma 2001;18:1229-1240.

15. Büki A, Siman R, Trojanowski JQ, Povlishock JT. The role of calpain-mediated spectrin proteolysis in traumatically induced axonal injury. J Neuropathol Exp Neurol 1999;58:365-375.

16. McGinn MJ, Kelley BJ, Akinyi L, et al. Biochemical, structural, and biomarker evidence for calpain-mediated cytoskeletal change after diffuse brain injury uncomplicated by contusion. J Neuropathol Exp Neurol 2009;68:241-249.

17. Huh JW, Franklin MA, Widing AG, Raghupathi R. Regionally distinct patterns of calpain activation and traumatic axonal injury following contusive brain injury in immature rats. Dev Neurosci 2006;28:466-476.

18. Kupina NC, Detloff MR, Bobrowski WF, Snyder BJ, Hall ED. Cytoskeletal protein degradation and neurodegeneration evolves differently in males and females following experimental head injury. Exp Neurol 2003;180:55-73.

19. Pike BR, Zhao X, Newcomb JK, et al. Regional calpain and caspase-3 proteolysis of $\alpha$-spectrin after traumatic brain injury. Neuroreport 1998;9:2437-2442.

20. Deng Y, Thompson BM, Gao X, Hall ED. Temporal relationship of peroxynitrite-induced oxidative damage, calpain-mediated cytoskeletal degradation and neurodegeneration after traumatic brain injury. Exp Neurol 2007;205:154-165.

21. Thompson SN, Gibson TR, Thompson BM, Deng Y, Hall ED. Relationship of calpain-mediated proteolysis to the expression of axonal and synaptic plasticity markers following traumatic brain injury in mice. Exp Neurol 2006;201:253-265.

22. DeRidder MN, Simon MJ, Siman R, Auberson YP, Raghupathi R, Meaney DF. Traumatic mechanical injury to the hippocampus in vitro causes regional caspase- 3 and calpain activation that is influenced by NMDA receptor subunit composition. Neurobiol Dis 2006;22:165-176.

23. Pike BR, Zhao X, Newcomb JK, Glenn CC, Anderson DK, Hayes RL. Stretch injury causes calpain and caspase- 3 activation and necrotic and apoptotic cell death in septo-hippocampal cell cultures. J Neurotrauma 2000;17:283-298.

24. McCracken E, Hunter AJ, Patel S, Graham DI, Dewar D. Calpain activation and cytoskeletal protein breakdown in the corpus callosum of head-injured patients. J Neurotrauma 1999;16:749-761.

25. Newcomb JK, Kampfl A, Posmantur RM, et al. Immunohisto- chemical study of calpain-mediated breakdown products to $\alpha$-spectrin following controlled cortical impact injury in the rat. J Neurotrauma 1997;14:369-383.

26. Huh JW, Widing AG, Raghupathi R. Basic science; repetitive mild non-contusive brain trauma in immature rats exacerbates traumatic axonal injury and axonal calpain activation: a preliminary report. J Neurotrauma 2007;24:15-27.

27. Maki M, Takano E, Mori $\mathrm{H}$, et al. All four internally repetitive domains of pig calpastatin possess inhibitory activities against calpains I and II. FEBS Lett 1987;223:174-180.

28. Hood JL, Logan BB, Sinai AP, Brooks WH, Roszman TL. Association of the calpain/calpastatin network with subcellular organelles. Biochem Biophys Res Commun 2003;310:1200-1212.

29. Garcia M, Bondada V, Geddes JW. Mitochondrial localization of mu-calpain. Biochem Biophys Res Commun 2005;338:12411247.

30. Tremper-Wells B, Vallano ML. Nuclear calpain regulates $\mathrm{Ca}^{2+}$ dependent signaling via proteolysis of nuclear $\mathrm{Ca}^{2+} /$ calmodulindependent protein kinase type IV in cultured neurons. J Biol Chem 2005;280:2165-2175.

31. Kilinc D, Gallo G, Barbee KA. Mechanical membrane injury induces axonal beading through localized activation of calpain. Exp Neurol 2009;219:553-561.

32. Tullio RD, Passalacqua M, Averna M, Salamino F, Melloni E, Pontremoli S. Changes in intracellular localization of calpastatin during calpain activation. Biochem J 1999;343 Pt 2:467-472.

33. Newcomb JK, Pike BR, Zhao X, Banik NL, Hayes RL. Altered calpastatin protein levels following traumatic brain injury in rat. J Neurotrauma 1999;16:1-11.

34. De Tullio R, Averna M, Stifanese R, et al. Multiple rat brain calpastatin forms are produced by distinct starting points and alternative splicing of the N-terminal exons. Arch Biochem Biophys 2007;465:148-156.

35. Foozer H, Surles N, Kizhakke Madathil S, Saatman K. Behavioral dysfunction and histological damage in calpastatin knockout mice after controlled cortical impact injury. J Neurotrauma 2007;24: 1250 (abstract).

36. Ringger NC, Tolentino PJ, McKinsey DM, Pike BR, Wang KK, Hayes RL. Effects of injury severity on regional and temporal mRNA expression levels of calpains and caspases after traumatic brain injury in rats. J Neurotrauma 2004;21:829-841.

37. Guttmann RP, Elce JS, Bell PD, Isbell JC, Johnson GV. Oxidation inhibits substrate proteolysis by calpain I but not autolysis. J Biol Chem 1997;272:2005-2012.

38. Hall ED, Vaishnav RA, Mustafa AG. Antioxidant therapies for traumatic brain injury. Neurotherapeutics 2010;7:51-61.

39. Roberts-Lewis JM, Savage MJ, Marcy VR, Pinsker LR, Siman R. Immunolocalization of calpain I-mediated spectrin degradation to vulnerable neurons in the ischemic gerbil brain. J Neurosci 1994; 14:3934-3944.

40. Saido TC, Yokota M, Nagao S, et al. Spatial resolution of fodrin proteolysis in postischemic brain. J Biol Chem 1993;268:2523925243.

41. Wang KK. Calpain and caspase: can you tell the difference? [Erratum in: Trends Neurosci 2000;23:59] Trends Neurosci 2000;23: $20-26$.

42. Taft WC, Yang K, Dixon CE, Hayes RL. Microtubule-associated protein 2 levels decrease in hippocampus following traumatic brain injury. J Neurotrauma 1992;9:281-290.

43. Franz G, Beer R, Intemann D, et al. Temporal and spatial profile of Bid cleavage after experimental traumatic brain injury. J Cereb Blood Flow Metab 2002;22:951-958.

44. von Reyn CR, Spaethling JM, Mesfin MN, et al. Calpain mediates proteolysis of the voltage-gated sodium channel $\alpha$-subunit. J Neurosci 2009;29:10350-10356

45. Saatman KE, Graham DI, McIntosh TK. The neuronal cytoskeleton is at risk after mild and moderate brain injury. J Neurotrauma 1998;15:1047-1058.

46. Posmantur RM, Hayes RL, Dixon CE, Taft WC. Neurofilament 68 and Neurofilament 200 protein levels decrease after TBI. J Neurotrauma 1994;11:533-545.

47. Serbest G, Burkhardt MF, Siman R, Raghupathi R, Saatman KE 
Temporal profiles of cytoskeletal protein loss following traumatic axonal injury in mice. Neurochem Res 2007;32:2006-2014.

48. Huh JW, Laurer HL, Raghupathi R, Helfaer MA, Saatman KE. Rapid loss and partial recovery of neurofilament immunostaining following focal brain injury in mice. Exp Neurol 2002;175:198208.

49. Posmantur RM, Zhao X, Kampfl A, Clifton GL, Hayes RL. Immunoblot analyses of the relative contributions of cysteine and aspartic proteases to neurofilament breakdown products following experimental brain injury in rats. Neurochem Res 1998;23:12651276.

50. Posmantur R, Kampfl A, Siman R, Liu J, Zhao X, Clifton GL, Hayes RL. A calpain inhibitor attenuates cortical cytoskeletal protein loss after experimental traumatic brain injury in the rat. Neuroscience 1997;77:875-888.

51. Liu MC, Akle V, Zheng W, et al. Extensive degradation of myelin basic protein isoforms by calpain following traumatic brain injury. J Neurochem 2006;98:700-712.

52. Zhang Z, Ottens AK, Sadasivan S, et al. Calpain-mediated collapsin response mediator protein-1, -2 , and -4 proteolysis after neurotoxic and traumatic brain injury. J Neurotrauma 2007;24:460472.

53. Liu MC, Akle V, Zheng W, et al. Comparing calpain- and caspase3 -mediated degradation patterns in traumatic brain injury by differential proteome analysis. Biochem J 2006;394:715-725.

54. Siman R, McIntosh TK, Soltesz KM, Chen Z, Neumar RW, Roberts VL. Proteins released from degenerating neurons are surrogate markers for acute brain damage. Neurobiol Dis 2004;16:311-320.

55. Hicks R, Soares H, Smith D, McIntosh T. Temporal and spatial characteristics of neuronal injury following lateral fluid-percussion brain injury in the rat. Acta Neuropathol 1996;91:236-246.

56. Fineman I, Hovda DA, Smith M, Yoshino A, Becker DP. Concussive brain injury is associated with a prolonged accumulation of calcium: a ${ }^{45} \mathrm{Ca}$ autoradiographic study. Brain Res 1993;624:94102.

57. Hall ED, Gibson TR, Pavel KM. Lack of a gender difference in post-traumatic neurodegeneration in the mouse controlled cortical impact injury model. J Neurotrauma 2005;22:669-679.

58. Farkas O, Lifshitz J, Povlishock JT. Mechanoporation induced by diffuse traumatic brain injury: an irreversible or reversible response to injury? J Neurosci 2006;26:3130-3140.

59. Hicks RR, Smith DH, Lowenstein DH, Saint Marie R, McIntosh TK. Mild experimental brain injury in the rat induces cognitive deficits associated with regional neuronal loss in the hippocampus. J Neurotrauma 1993;10:405-414.

60. Beer R, Franz G, Srinivasan A, et al. Temporal profile and cell subtype distribution of activated caspase- 3 following experimental traumatic brain injury. J Neurochem 2000;75:1264-1273.

61. Clark RSB, Kochanek PM, Watkins SC, et al. Caspase-3 mediated neuronal death after traumatic brain injury in rats. J Neurochem 2000;74:740-753.

62. Yakovlev AG, Knoblach SM, Fan L, Fox GB, Goodnight R, Faden AI. Activation of CPP32-like caspases contributes to neuronal apoptosis and neurological dysfunction after traumatic brain injury. J Neurosci 1997;17:7415-7424.

63. Raghupathi R. Cell death mechanisms following traumatic brain injury. Brain Pathol 2004;14:215-222.

64. Saatman KE, Duhaime AC, Bullock R, Maas AI, Valadka A, Manley GT; Workshop Scientific Team and Advisory Panel Members. Classification of traumatic brain injury for targeted therapies. J Neurotrauma 2008;25:719-738.

65. Pineda JA, Wang KK, Hayes RL. Biomarkers of proteolytic damage following traumatic brain injury. Brain Pathol 2004;14:202209.

66. Ottens AK, Golden EC, Bustamante L, Hayes RL, Denslow ND, Wang KK. Proteolysis of multiple myelin basic protein isoforms after neurotrauma: characterization by mass spectrometry. J Neurochem 2008;104:1404-1414.

67. Pike BR, Flint J, Dutta S, Johnson E, Wang KK, Hayes RL. Accumulation of non-erythroid $\alpha$ II-spectrin and calpain-cleaved $\alpha$ II-spectrin breakdown products in cerebrospinal fluid after traumatic brain injury in rats. J Neurochem 2001;78:1297-1306.

68. Aikman J, O'Steen B, Silver X, et al. $\alpha$-II-spectrin after controlled cortical impact in the immature rat brain. Dev Neurosci 2006;28:457-465.

69. Ringger NC, O'Steen BE, Brabham JG, et al. A novel marker for traumatic brain injury: CSF $\alpha$ II-spectrin breakdown product levels. J Neurotrauma 2004;21:1443-1456.

70. Farkas O, Polgár B, Szekeres-Barthó J, Dóczi T, Povlishock JT, Büki A. Spectrin breakdown products in the cerebrospinal fluid in severe head injury: preliminary observations. Acta Neurochir (Wien) 2005;147:855-861.

71. Pineda JA, Lewis SB, Valadka AB, et al. Clinical significance of $\alpha$ II-spectrin breakdown products in cerebrospinal fluid after severe traumatic brain injury. J Neurotrauma 2007;24:354-366.

72. Cardali S, Maugeri R. Detection of $\alpha \mathrm{II}$-spectrin and breakdown products in humans after severe traumatic brain injury. J Neurosurg Sci 2006;50:25-31.

73. Brophy GM, Pineda JA, Papa L, et al. $\alpha$ II-Spectrin breakdown product cerebrospinal fluid exposure metrics suggest differences in cellular injury mechanisms after severe traumatic brain injury. J Neurotrauma 2009;26:471-479.

74. Cox CD, West EJ, Liu MC, Wang KK, Hayes RL, Lyeth BG Dicyclomine, an M1 muscarinic antagonist, reduces biomarker levels, but not neuronal degeneration, in fluid percussion brain injury. J Neurotrauma 2008;25:1355-1365.

75. Haranishi Y, Kawata R, Fukuda S, et al. Moderate hypothermia, but not calpain inhibitor 2, attenuates the proteolysis of microtubule-associated protein 2 in the hippocampus following traumatic brain injury in rats. Eur J Anaesthesiol 2005;22:140-147.

76. Saatman KE, Murai H, Bartus RT, et al. Calpain inhibitor AK295 attenuates motor and cognitive deficits following experimental brain injury in the rat. Proc Natl Acad Sci U S A 1996;93:3428-3433.

77. Saatman KE, Zhang C, Bartus RT, McIntosh TK. Behavioral efficacy of posttraumatic calpain inhibition is not accompanied by reduced spectrin proteolysis, cortical lesion, or apoptosis. J Cereb Blood Flow Metab 2000;20:66-73.

78. Lubisch W, Beckenbach E, Bopp S, et al. Benzoylalanine-derived ketoamides carrying vinylbenzyl amino residues: discovery of potent water-soluble calpain inhibitors with oral bioavailability. J Med Chem 2003;46:2404-2412.

79. Markgraf CG, Velayo NL, Johnson MP, et al. Six-hour window of opportunity for calpain inhibition in focal cerebral ischemia in rats. Stroke 1998;29:152-158.

80. Buki A, Farkas O, Doczi T, Povlishock JT. Preinjury administration of the calpain inhibitor MDL-28170 attenuates traumatically induced axonal injury. J Neurotrauma 2003;20:261-268.

81. Ai J, Liu E, Wang J, Chen Y, Yu J, Baker AJ. Calpain inhibitor MDL-28170 reduces the functional and structural deterioration of corpus callosum following fluid percussion injury. J Neurotrauma 2007;24:960-978.

82. Guttmann RP, Baker DL, Seifert KM, Cohen AS, Coulter DA, Lynch DR. Specific proteolysis of the NR2 subunit at multiple sites by calpain. J Neurochem 2001;78:1083-1093.

83. Saatman KE, Bareyre FM, Grady MS, McIntosh TK. Acute cytoskeletal alterations and cell death induced by experimental brain injury are attenuated by magnesium treatment and exacerbated by magnesium deficiency. J Neuropathol Exp Neurol 2001;60:183-194.

84. Liu L, Yenari MA. Therapeutic hypothermia: neuroprotective mechanisms. Front Biosci 2007;12:816-825.

85. Büki A, Koizumi H, Povlishock JT. Moderate posttraumatic hypothermia decreases early calpain-mediated proteolysis and concomitant cytoskeletal compromise in traumatic axonal injury. Exp Neurol 1999;159:319-328.

86. Temkin NR, Anderson GD, Winn HR, et al. Magnesium sulfate for neuroprotection after traumatic brain injury: a randomised controlled trial. Lancet Neurol 2007;6:29-38.

87. Clifton GL, Miller ER, Choi SC, et al. Lack of effect of induction of hypothermia after acute brain injury. N Engl J Med 2001;344: 556-563.

88. Pettus EH, Christman CW, Giebel ML, Povlishock JT. Traumatically induced altered membrane permeability: its relationship to traumatically induced reactive axonal change. J Neurotrauma 1994;11:507-522.

89. Deng-Bryant Y, Singh IN, Carrico KM, Hall ED. Neuroprotective effects of tempol, a catalytic scavenger of peroxynitrite-derived 
free radicals, in a mouse traumatic brain injury model. J Cereb Blood Flow Metab 2008;28:1114-1126.

90. Okonkwo DO, Büki A, Siman R, Povlishock JT. Cyclosporin A limits calcium-induced axonal damage following traumatic brain injury. Neuroreport 1999;10:353-358.

91. Büki A, Okonkwo DO, Povlishock JT. Postinjury cyclosporin A administration limits axonal damage and disconnection in traumatic brain injury. J Neurotrauma 1999;16:511-521.
92. Mbye LH, Singh IN, Carrico KM, Saatman KE, Hall ED. Comparative neuroprotective effects of cyclosporin A and NIM811, a nonimmunosuppressive cyclosporin A analog, following traumatic brain injury. J Cereb Blood Flow Metab 2009;29:87-97.

93. Kupina NC, Detloff MR, Dutta S, Hall ED. Neuroimmunophilin ligand V-10,367 is neuroprotective after 24-hour delayed administration in a mouse model of diffuse traumatic brain injury. J Cereb Blood Flow Metab 2002;22:1212-1221. 